MARITA MEYER

\title{
Deutsche und polnische Lektüren über den Zwei- ten Weltkrieg und den Holocaust. Eine vergleichende Untersuchung zur schulischen Kanonbildung und zum kulturellen Gedächtnis
}

Artykuł jest próbą porównania polskich i niemieckich lektur szkolnych dotyczących tematyki II Wojny Światowej i Holocaustu. Ponieważ po stronie niemieckiej ściśle określony kanon lektur nie istnieje, konieczne okazało się przeprowadzenie ankiety umożliwiającej ustalenie kanonu ,tajemnego‘. Po stronie polskiej obowiązują nie tylko ustalone lektury, lecz również, aż do egzaminu dojrzałości, podręczniki służące nauczaniu literatury. Celem przeprowadzonego badania jest porównanie opracowań na temat należących do kanonu lektur autorów (Tadeusz Borowski, Czesław Miłosz, Hanna Krall) w trzech polskich podręcznikach szkolnych.

Der Aufsatz versucht die polnischen und die deutschen Schullektüren zum Thema Zweiter Weltkrieg und Holocaust miteinander zu vergleichen. Da auf deutscher Seite kein festgelegter Kanon existiert, war eine Umfrage notwendig, um den ,heimlichen Kanon zu ermitteln. Auf der polnischen Seite gibt es nicht nur festgelegte Lektüren, sondern bis zum Abitur auch Schulbücher im Literaturunterricht. Ein Vergleich dreier polnischer Schulbücher und ihrer Behandlung der kanonischen Autoren (Tadeusz Borowski, Czesław Miłosz, Hanna Krall) steht im Zentrum der Untersuchung.

This article tries to compare books read at schools in Germany and in Poland concerning Second World War und Holocaust. As there isn't a fixed canon of books for German schools, an inquiry became necessary to find out the ,secret ' canon. In Poland there are not only established compulsory books but also textbooks are used in preparation to matriculation. An investigation of three Polish textbooks and the canonical authors (Tadeusz Borowski, Czesław Miłosz, Hanna Krall) is in the centre of this article. 
Marita Meyer

„Im Grunde genommen gab es ebenso viele Zweite Weltkriege wie Nationen“, schrieb der Publizist ADAM KRZEMIŃSKI (2005) in der polnischen Zeitschrift Polityka. Anfang und Ende des Krieges, Verlauf und Perspektiven der einzelnen Nationen sind so unterschiedlich, dass eine gemeinsame europäische Erzählung darüber kaum je möglich zu sein scheint. Voraussetzung für jede mögliche Verständigung auf eine supranationale Perspektive ist die Kenntnis der Perspektive der Nachbarn. Mit Blick auf die Nachbarn Deutschland und Polen muss man sagen, dass das gegenseitige Unwissen noch immer groß ist. Die kollektiven Gedächtnisse haben jeweils unterschiedliche Ereignisse festgehalten und verschiedene wirkungsmächtige Symbole und Texte in den Mittelpunkt der nationalen Erinnerung gestellt. Als Beispiel für das Unwissen auf deutscher Seite sei angeführt, dass die deutsche Geschichtsforschung bis vor kurzem davon ausging, dass der Vernichtungskrieg im Jahr 1942 begann. Über 60 Jahre nach Kriegsende wies der Historiker JoCHEN BÖHLER (2006) nach, dass die deutsche Kriegsführung bereits 1939 mit dem Überfall auf Polen auf Vernichtung angelegt war. Verantwortlich für die Wissenslücken sind neben Verdrängung auch Sprachbarrieren und die lang andauernde ideologische Trennung. Andererseits bewirkte die politische Zensur auf der polnischen Seite lange keine befriedigende Selbstauskunft über die Ereignisse im eigenen Land. Als Stichworte seien nur die Widerstandsbewegung genannt, die sich nicht nur gegen die deutschen, sondern auch gegen die sowjetischen Besatzer richtete, und deren Thematisierung nicht opportun war, genauso wenig wie die des Antisemitismus; von der zunächst ungleichzeitigen und danach gegenläufig geführten Debatte über die Vertreibungen ganz zu schweigen. Der Blick dieser Untersuchung richtet sich auf die literarische Verarbeitung eines Teils dieser Vergangenheit: die Massenverbrechen der Nationalsozialisten, denen in Polen Juden und Nichtjuden zum Opfer fielen. Wenn hier der Begriff ,Holocaust ' benutzt wird, dann ist damit nicht ausschließlich die Judenvernichtung gemeint, sondern die gezielte und industriell durchgeführte Vernichtung aller von der nationalsozialistischen Ideologie als minderwertig eingestuften Menschengruppen. Auch mag der Begriff eine problematische Etymologie haben, seine Herkunft aus dem griechischen Wort für ,Brandopfer', sein Gebrauch aber ist zunehmend verbreitet und löst m.E. in der Regel zutreffende Assoziationen aus. Auch in Polen wird er immer häufiger neben dem polnischen Wort für Vernichtung, ,zagłada', benutzt. 
In den Diskussionen über ein europäisches Gedächtnis spielt der Vorschlag von Dan Diner, den Holocaust zu einem gemeinsamen Ausgangspunkt der Erinnerungspolitik zu machen, mittlerweile eine wichtige Rolle. ${ }^{1}$ Dabei hat der Holcoaust als gemeinsames Gedächtnis, speziell zwischen Polen und Deutschland, mindestens zwei problematische Aspekte. Einerseits droht in Deutschland immer wieder mal das Opfergedächtnis das Tätergedächtnis zu verdrängen, andererseits gibt es in Polen die Befürchtung, dass eine auf den Holocaust als Judenvernichtung festgelegte Erinnerung die Verbrechen an den Polen insgesamt noch mehr ausblende als bisher schon. Bisher stehen vor allem der Geschichtsunterricht und damit die historischen Lehrbücher im Mittelpunkt des Interesses, wenn es um die Untersuchung von Unterschieden und um Versuche von Dialog und Annäherung geht. Die Empfehlungen der deutsch-polnischen Schulbuchkommission haben dazu beigetragen, dass sich seit den 90er Jahren auf beiden Seiten Fortschritte zeigen. So wurden die polnischen Geschichtsbücher in der Darstellung des Zweiten Weltkriegs differenzierter (ANNA LANDAU-CZAJKA 2005). Und in den deutschen Geschichtsbüchern wurden die Beiträge über Polen umfangreicher und zugleich klarer (WŁODZIMIERZ BORODZIEJ 2005). Vergleichende Betrachtungen zum Literaturunterricht der beiden Länder fehlen dagegen, obschon literarische Werke in besonderer Weise das kulturelle Gedächtnis prägen, besonders wenn sie über eine Kanonisierung Funktionen in diesem Gedächtnis einnehmen. Welche Werke werden in deutschen und in polnischen Schulen zum Thema Nationalsozialismus und Holocaust gelesen? Gibt es kanonische Werke, die die Vorstellungswelt über die Verbrechen des Zweiten Weltkrieges besonders prägen? Sind diese Werke in beiden Ländern vergleichbar? Wo liegen Gemeinsamkeiten und Unterschiede? Barbara Breysach hat sich sowohl mit der polnischen als auch mit der deutschsprachigen Literatur über die Vernichtung ausführlich auseinandergesetzt. Sie konstatiert ein ausgeprägtes „Nebeneinander der Gedächtnisdeterminanten“ (BREYSACH 2005: 400) und hat sich wohl auch wegen dieser Asymmetrien der polnischen und der deutschsprachigen Literatur mit jeweils unterschiedlichen Fragestellungen genähert. Die folgende Untersuchung beschränkt sich auf die schulischen Lektüren. Das bedeutet eine Beschränkung auf einen Korpus von Texten, der allerdings besonders einflussreich ist. Die Entscheidungen für oder gegen Schullektüren sind Teil auch kulturpolitisch relevanter Entwicklungen. Der Zweite Weltkrieg im Allgemeinen und der Holocaust im Besonderen sind

1 Vgl. dazu die Überlegungen Aleida Assmanns zu Europa als Erinnerungsgemeinschaft. In: Der lange Schatten der Vergangenheit (2006:255-258). 
Marita Meyer

heute in beiden Ländern Schulstoff. Man sollte annehmen, dass es verallgemeinerbare Erfahrungen und Erkenntnisse über diese Zeit und ihre Folgen gibt. Bei aller Asymmetrie wird eine Vergleichbarkeit daher nicht ausgeschlossen.

\section{Der ,heimliche‘ Kanon in Deutschland}

Schon am Beginn eines Vergleichs steht allerdings eine solche Asymmetrie: die methodische Schwierigkeit, dass es in Deutschland anders als in Polen kein zentrales Abitur und keinen festgelegten Lektürekanon gibt. Seit den 70er Jahren werden in Westdeutschland (seit den 90er Jahren im gesamten Land) lediglich Vorgaben zu Epochen und Gattungen im Lehrplan gemacht, über die einzelnen Lektüren entscheidet jeder Lehrer eigenständig. Somit wurde eine Umfrage notwendig, um zu ermitteln, ob es trotz fehlendem offiziellen Kanon in Deutschland einen ,heimlichen“ Kanon von besonders häufig gelesenen Werken gibt und wenn ja, um welche Werke es sich handelt. Bisher lag nur eine umfassende Untersuchung zu den Lektüren an den Schulen des Landes Nordrhein-Westfalen vor, die allerdings von 1992 stammte und nur die Sekundarstufe 1 betraf (KULTUSMINISTERIUM NRW 1994). Da in Polen das Thema Holocaust aber ein Jahr vor dem Abitur (,matura') behandelt wird, waren auch für Deutschland vor allem die Lektüren der Sekundarstufe 2 (Klassen 11 bis 13) von Interesse. Es wurde also im Sommer und Herbst 2007 eine schriftliche Umfrage unter Berliner Studierenden über ihre schulischen Lektüren durchgeführt (insgesamt konnten 227 Fragebögen ausgewertet werden). Außerdem wurde eine Kontrollstudie unter Berliner Deutschlehrern durchgeführt. Auf die genaue Darstellung und die detaillierte Auswertung der Umfrage wird an dieser Stelle verzichtet. ${ }^{2}$ Allein das Ergebnis ist hier als Grundlage für vergleichende Überlegungen von Interesse. Die Umfrage ergab, dass trotz Verzicht auf einen offiziellen Lektürekanon ein sogenannter ,heimlicher' oder impliziter Lektürekanon existiert, d.h. einige wenige Bücher werden besonders häufig und immer wieder gelesen. Das Ergebnis für die Sekundarstufe 1 bestätigte das Ergebnis der NRW-Befragung von 1992: Am häufigsten wurde Das Tagebuch der Anne Frank genannt, außerdem Damals war es Friedrich von Hans Peter Richter, Als Hitler das rosa Kaninchen stahl von Judith Kerr und Die Welle von Morton Rhue

2 Darstellung und Auswertung der Umfragen samt Grafiken wurden in der Zeitschrift Der Deutschunterricht veröffentlicht, vgl. MEYER (2008:74-80). 
(engl. Originaltitel: The wave). Bei der NRW-Befragung kam außerdem Der gelbe Vogel von Myron Levoy (engl. Originaltitel: Alan and Naomi), in die Spitzengruppe, ein Titel, der in Berlin nur einige Male genannt wurde. In allen Fällen handelt es sich um Werke der Kinder- und Jugendliteratur. Der Holocaust im eigentlichen Sinn kommt in diesen Werken nicht vor, Thema sind meistens die Verfolgung in der NS-Zeit oder die Nachwirkungen der Verbrechen. Für die Sekundarstufe 2, die Stufe, die uns besonders interessiert, gab es einen Titel, der mit Abstand am häufigsten genannt wurde: Der Vorleser, ein Roman von Bernhard Schlink. Außerdem wurden das Gedicht Todesfuge von Paul Celan häufig genannt, der Roman Jakob der Lügner von Jurek Becker und der Roman Das siebte Kreuz von Anna Seghers. Die Kontrollstudie unter Lehrenden bestätigte dieses Ergebnis.

Bereits die Übereinstimmungen mit der Befragung von 1992 deuten auf Kontinuität im Kanon hin, was ja gerade die Abschaffung des festgelegten Kanons mindern sollte. Es sollte gewährleistet werden, dass Werke nicht aus Gewohnheit als Lektüre gewählt werden, sondern bewusst und unter Umständen auch gegen aktuellere und jeweils geeignetere Werke ausgetauscht werden. Die Kontinuität des Kanons deutet weniger auf die gewünschte „Arbeit am Kanon“ (FINGERHUT 1994:51) hin, als darauf, dass „,das Verläßlichste der Kanon von gestern“ ist (MÜLLER-MICHAELS 1994:54). Ein weiteres Indiz für Kontinuität sind auch die Unterschiede zwischen östlichen und westlichen Bundesländern in der Umfrage. Studierende, die in den alten Bundesländern Abitur gemacht hatten, hatten vermehrt das Buch des jüdischen DDRDissidenten Jurek Becker genannt, während in den östlichen Bundesländern Das siebte Kreuz, ein Roman über verfolgte Kommunisten, gelesen wurde. Gerade entlang der politischen Denkgewohnheiten aus den Zeiten der Teilung hätte man mehr Bewegung im Kanon erwarten können. Ein weiteres überraschendes Ergebnis der Umfrage - vor allem aus literaturwissenschaftlicher Sicht - ist die Kluft zwischen Literaturwissenschaft und schulischer Praxis. Am deutlichsten ist der Unterschied im Fall von Schlinks Vorleser. Der Roman wurde von der Literaturkritik zunächst überwiegend positiv besprochen und wurde auch zu einem Bestseller. Erst nach einer scharfen Kritik des englischen Literaturwissenschaftlers Jeremy Adler in der Süddeutschen Zeitung (20.4.2002) meldeten sich vermehrt auch kritische deutsche Stimmen zu Wort. In einschlägigen Werken der Literaturwissenschaft wird der Roman entweder ignoriert (BRAESE 1998, 1998a; EKE / STEINECKE 2006) oder wenn behandelt, dann meistens kritisch besprochen (HOFMANN 2003; SCHLANT 
Marita Meyer

2001; HAHN 2005). ${ }^{3}$ Da Schlinks Roman mittlerweile in der polnischen Germanistik als Lektüre eine gewisse Verbreitung gefunden hat, soll hier kurz auf seine Problematik eingegangen werden. Der Roman zeichnet sich wie bereits die zuvor von Schlink verfassten Krimis durch einen spannungsreichen Plot und leichte Lesbarkeit aus, was sicher eine Erklärung für den Erfolg des Buches bei einem breiten Publikum ist. Eine andere Erklärung ist die Wahl einer ungewöhnlichen und durchaus interessanten Erzählperspektive in der Behandlung der deutschen Nazi-Vergangenheit. Es wird aus der Sicht eines Angehörigen der zweiten Generation erzählt, der sich als Jugendlicher in eine erwachsene Frau verliebt, ohne zu wissen, dass sie während des Kriegs als Aufseherin in einem Konzentrationslager war. Als Jurastudent sieht er seine ehemalige Geliebte als Angeklagte in einem der Auschwitzprozesse wieder. Die Schwächen des Romans bestehen in der nur unbefriedigend eingelösten Behauptung, die Vergangenheit aufzuarbeiten. So erfährt man nie Genaues über die Rolle der weiblichen Hauptfigur namens Hanna im Konzentrationslager. Zudem wird ihr Analphabetentum zu einem entschuldigenden Motiv. Sie bekommt Züge eines Opfers, das in die Rolle als Täterin nur , hineingerutscht ' ist. In der Darstellung der wirklichen Opfer dagegen fehlt es dem Roman an Einfühlungsvermögen. Und schließlich wirkt die Läuterung Hannas im Gefängnis durch Lektüre von Holocaustliteratur arg konstruiert. Allerdings wurde mit der vielfachen Entscheidung für diesen Roman aus dem Jahr 1995 als Schullektüre tatsächlich die Kontinuität des tradierten Kanons durchbrochen. Auch könnte man einwenden, dass die Wahl der Lektüre noch wenig über ihre Behandlung im Unterricht aussagt. Ein Blick in die verschiedenen Unterrichtshilfen, die mittlerweile zum Roman erschienen sind, stimmt aber nicht optimistisch, was die Verbreitung von kritischen Lesarten betrifft. ${ }^{4}$

3 Eine Ausnahme stellt die verteidigende Darstellung von OSTERMANN (2004) dar.

Die meisten Materialien beschränken sich auf formale Beschreibungen und inhaltliche Zusammenfassungen. Hier seien nur zwei Interpretationshilfen erwähnt, die neben ihrer wohlwollenden Gesamtdeutung auch vereinzelt Schwächen feststellen: Juliane KöSTER (2005:64) sieht ein Dilemma im Versuch, das Verhalten Hannas zu verstehen und gleichzeitig zu verurteilen. (Würde es sich hier wirklich um ein unauflösbares Dilemma handeln, wie sollten Richter ihren Beruf ausüben?). Zwar werde Hanna im Roman verurteilt, die affektiven Leseangebote aber würden diese Verurteilung unterlaufen. SASCHA FEUCHERT und LaRs Hofmann (2005:38) sehen die Schwächen des Romans in den Deformierungen des Erzählers begründet. Es sei Aufgabe des Lesers, die unzureichenden und falschen Schlussfolgerungen der Erzählerfigur zu korrigieren (FEUCHERT / 
Ein weiteres Indiz für das Auseinanderklaffen von Literaturwissenschaft und schulischer Praxis sind die seltenen Nennungen von anerkannten Werken der Weltliteratur oder auch von deutschsprachigen Werken, die neben der Weltliteratur bestehen können. So wurde Die Ermittlung von Peter Weiss nur viermal genannt, weiter leben von Ruth Klüger kein einziges Mal. Bei der Todesfuge besteht diese Kluft nicht, versteht sich. Auch Das siebte Kreuz und Jakob der Lügner sind literarisch anerkannte Werke. Allerdings behandelt der Roman von Anna Seghers die Zeit der Verfolgung, die größeren Verbrechen der Kriegszeit konnte der 1937 geschriebene Roman noch nicht thematisieren, und Jurek Beckers Roman kann durch seinen humorvoll-heiteren Tonfall durchaus zu einer entlastenden Lesart verleiten. Nicht unerwähnt bleiben soll, dass vereinzelt die Möglichkeiten der freien Lektürewahl auch für sehr ambitionierte Lektüren genutzt werden. So wurde zum Beispiel bei der Umfrage zweimal Roman eines Schicksallosen von Imre Kertész genannt. Bei der Suche nach den besonders weit verbreiteten Lektüren fielen diese vereinzelten Nennungen nicht ins Gewicht.

\section{Der offizielle Kanon in Polen und seine Behandlung in Schulbüchern}

Anders als in Deutschland gibt es in Polen einen Kanon von Autoren und Werken, die bis zum Abitur gelesen werden müssen. Auch zum Thema „Holocaust“ sind bestimmte Werke als Lektüre vorgeschrieben. Dieser Kanon beinhaltet seit langem schon die Erzählungen von Tadeusz Borowski, in den 90er Jahren kamen die Gedichte des zuvor zensierten Czesław Miłosz sowie Dem Herrgott zuvorkommen (Zdażyc przed Panem Bogiem) von Hanna Krall hinzu. Zofia Nałkowskas Prosastücke Medaillons (Medaliony) gehörten noch in den 90er Jahren zum Kanon, heute jedoch nicht mehr, wer-

HofmanN 2005:38). Diese Lesart besitzt Züge einer ,rettenden“ Kritik für den Roman. Sie setzt allerdings ein profundes Vorwissen über die vergangenheitspolitischen und ästhetischen Diskurse beim Leser voraus. An dieser Stelle sei auf die Verfilmung des Romans durch Stephen Daldry (2008) hingewiesen. Interessanterweise ist in The reader der Versuch erkennbar, einige Schwächen des Romans mit filmischen Mitteln quasi zu korrigieren. Dazu gehört, den (erwachsenen) Erzähler anders als im Roman von Beginn an als emotional beschädigt einzuführen sowie das Weglassen zentraler unzulänglicher Reflexionen des Erzählers. An die Stelle dieser Reflexionen tritt der Blick einer Kamera, der stärker als es im Roman geschieht, Einfühlung in die Situation der überlebenden jüdischen Opfer einfordert. 
den gleichwohl noch von vielen Lehrern gern als zusätzliche Lektüre gewählt, so dass dieser Text vielen polnischen Studierenden bekannt ist und als kanonisch im impliziten Sinne gelten kann. Ebenfalls von Studierenden häufig genannt werden Gespräche mit dem Henker (Rozmowy z katem) von Kazimierz Moczarski. Hierin erinnert sich der Journalist Moczarski an seine Gespräche mit Jürgen Stroop, mit dem er mehrere Jahre eine Zelle in einem Warschauer Gefängnis teilte. Moczarski war als Mitglied der im Untergrund kämpfenden Armia Krajowa (,Heimatarmee') inhaftiert (deren Widerstand sich auch gegen die russischen Besatzer richtete), Stroop als General der SS und Kommandant des Warschauer Ghettos. Dieses gewiss einzigartige Zeugnis wird hier genauso wenig betrachtet wie die sprachlich beeindruckenden Texte von Zofia Nałkowska. Im Mittelpunkt werden die Werke des aktuellen polnischen Lektürekanons stehen, also Werke von Borowski, Miłosz und Krall.

Mit der Kanonisierung einher geht die Verwendung von Lehrbüchern, auch im Literaturunterricht der höheren Klassen. Es sind vor allem drei Lehrwerke neueren Datums, die wahlweise verwendet werden und die hier helfen sollen, einen Eindruck von der Art und Weise der Vermittlung der kanonischen Werke zu geben: das von ANDRZEJ Z. MAKOWIECKI, ANDRZEJ MARKOWSKI, WŁODZIMIERZ PASZYŃSKI und TOMASZ WROCZYŃSKI (2004) herausgegebene „Pamiętajcie o ogrodach... “ („Erinnert euch an die Gärten...“, titelgebende Zeile eines Gedichts von Jonasz Kofta, im Folgenden als Pamiętajcie zitiert), das von Witold BobiŃski, ANNA JANUS-SitARZ und BOGUSŁAW KoŁCZ (2004) herausgegebene Barwy epok [Farben der Epochen] und das von ZBIGNIEW MAJCHROWSKI und STANISŁAW ROSIEK (2006) herausgegebene Między tekstami [Zwischen den Texten]. In allen drei Lehrwerken werden - wie zu erwarten ist - die kanonischen Werke behandelt, in zweien einige Auszüge aus Moczarskis Dokumentation, und nur in Pamiętajcie werden die Medaillons von Nałkowska kurz behandelt.

\section{Tadeusz Borowski}

Von den kanonischen Autoren zum Thema Holocaust ist Tadeusz Borowski derjenige, dem in allen drei Lehrwerken der meiste Platz eingeräumt wird. Zur Pflichtlektüre gehören die Erzählungen Abschied von Maria, Bei uns in Auschwitz, Bitte, die Herrschaften zum Gas und Schlacht bei Grunwald. Die Fragen und Aufgaben in den Schulbüchern konzentrieren sich besonders auf zwei dieser Erzählungen, auf Bei uns in Auschwitz und Bitte, die Herrschaf- 
ten zum Gas. Beides sind zweifelsohne zentrale Erzählungen im Werk Borowskis. Sie sind wichtige Berichte eines Augenzeugen über das Innere der Konzentrationslager. Und sie zeugen gleichzeitig von der großen literarischen Begabung ihres Autors. In seiner Rede zur Verleihung des Nobelpreises hat Imre Kertész die Erzählungen Borowskis hervorgehoben: „Nach authentischen Quellen suchend, las ich zum erstenmal die klaren, selbstquälerisch gnadenlosen Erzählungen Tadeusz Borowskis, darunter ,Bitte, die Herrschaften zum Gas!““ (KERTÉSZ 2003:250) Auch RUTH KLÜGER (1992:105) verweist in ihrer Autobiographie weiter leben auf Borowski, ,ein genialer junger Pole, der nach dem Krieg den Kopf in den Gasofen steckte, nachdem er den Gaskammern entgangen war". Für sie hat Borowski in Bei uns in Auschwitz besonders deutlich gemacht, welche fatale Rolle die Hoffnung im Lager spielte. Zum Thema Hoffnung sei auch hier aus Borowskis Erzählung zitiert:

Die Hoffnung läßt sie um jeden Tag Leben kämpfen, denn vielleicht bringt ja schon der nächste Tag die Befreiung. Vielleicht nicht einmal die Hoffnung auf eine andere, bessere Welt, sondern bloß noch die Hoffnung auf ein Leben, in dem es Ruhe und Frieden gibt. In der ganzen Menschheitsgeschichte war die Hoffnung im Menschen nie stärker, aber nie hat sie auch soviel Unheil angerichtet wie in diesem Krieg, wie in diesem Lager. Wir haben nicht gelernt, der Hoffnung zu entsagen, und deshalb sterben wir im Gas. (BOROwSKI 2006:44)

Eng mit der Hoffnung ist auch der Wunsch nach Normalität verbunden bzw. die menschliche Neigung, sich auch in elendsten Zuständen einzurichten, als seien sie die Normalität. So versucht man selbst an diesem Ort, sich damit zu trösten, dass es Schlimmeres gebe, etwa Birkenau (BOROwSKI 2006:16). Wer überleben will, darf sich vom massenhaften Morden und Sterben ringsum nicht so stark erschüttern lassen, dass die Kraft zum Weiterleben verloren geht. So entwickeln sich auch Gleichgültigkeit und Härte als Selbstschutz bei vielen Häftlingen. Demoralsierung, so bemerkt der Leser mitunter mit Verstörung, hat auch den Erzähler erreicht. Zu Recht hat KAROL SAUERLAND (2007:14) betont, dass dieser Ich-Erzähler kein autobiographischer Erzähler ist, wie manche Rezensenten meinten. Borowskis erzählerische Leistung besteht vor allem darin, seinen Erzähler in präzisem bis gleichgültigem, manchmal zynischem Tonfall vom Lager berichten zu lassen und gleichzeitig beim Leser das Mitgefühl für die Leidenden und Sterbenden wachzuhalten. Dem Autor Borowski ist viel zu sehr an der Darstellung von Demoralisierung gelegen, als dass man annehmen könnte, er sei selbst davon in besonderem Maße geprägt worden. Allerdings gehört es zum selbstquälerischen und schonungslosen Vorgehen Borowskis, dass er selbst immer wieder den Ich-Erzähler in 
die Nähe des autobiographischen Ichs bringt. Wie Borowski selbst ist der Ich-Erzähler ein Funktionshäftling mit Privilegien. Der Ich-Erzähler in Bitte, die Herrschaften zum Gas ist im sogenannten Kanada-Kommando tätig, was bedeutet, dass er die Aufgabe hat, Gepäck und Kleidung der Ankommenden zu sammeln und zu sortieren. Dabei fällt immer etwas für die Helfer ab, womit sie ihren Hunger stillen oder mit den Wachmannschaften handeln können.

An einem Beispiel soll das erzählerische Vorgehen Borowskis verdeutlicht werden: Ein Extrem der Brutalität stellt „ein Mann in grüner Uniform, mit mehr Silber überhäuft als die anderen“, dar, ein SS-Mann, er „verzieht angewidert das Gesicht", als ein neuer Transport mit Waggons eintrifft, aus denen Schreien und Röcheln dringt. Damit Ruhe einkehrt, befiehlt er, den Waggon mit der Maschinenpistole zu durchlöchern. Zu den Funktionshäftlingen gewendet sagt er: „Wer Gold oder sonst etwas nicht Essbares nimmt, wird als Dieb von Reichseigentum erschossen. Verstanden? ,Jawohl!‘ brüllt die Menge uneinheitlich und individuell, aber guten Willens." (BOROWSKI 2006:201) In diesem Zusammenhang wirkt schon der Ausdruck ,guten Willens" sarkastisch. Allerdings ist durch den Kontrast des äußerst brutalen und skrupellosen SS-Mannes ein deutlicher Unterschied zu den aus Not zum Mittun gezwungenen Häftlingen präsent. Noch deutlicher wird die demoralisierende Wirkung des Lagers bei der Schilderung der Verachtung, die manche Gefangene den hungernden Griechen im Lager entgegenbringen:

„Das sind Tiere. Morgen wird die Hälfte von ihnen an Dünnschiß krepieren.“ „Tiere? Du hast auch mal Hunger gehabt.“ „Tiere“, wiederhole ich verbissen. Ich schließe die Augen, höre die Schreie, spüre das Beben der Erde und die schwüle Luft auf meinen Augenlidern. Meine Kehle ist ganz ausgedörrt. (BoROWSKI 2006:208)

Die verächtliche Aussage des Ich-Erzählers über die hungernden Griechen wird zum einen durch den kritischen Kommentar des Mithäftlings gebrochen, zum anderen wird durch Formulierungen wie ,verbissen“ und „,spüre das Beben der Erde“ die innere Verfasstheit des Erzählers deutlich: als ständiger Kampf zwischen Erschütterung und Selbstbehauptung. Die Erzählung beschreibt wie kaum eine andere innerhalb der gesamten Holocaustliteratur das Verbrechen, Menschen zu Zeugen und zu Handlangern des Mordens zu machen, bis sie zuletzt Abscheu vor sich selbst entwickeln. Schließlich fühlt sich auch der so abgebrüht wirkende Ich-Erzähler einem weiteren Einsatz an der Rampe nicht mehr gewachsen.

Borowski versäumt nicht, darauf hinzuweisen, dass es nicht nur das Dasein als Funktionshäftling ist, das ihn selbst ebenso wie seine Erzähler vor dem 
sicheren Tod bewahrt, sondern dass er vor allem als Nichtjude in der Lagerhierarchie nicht an unterster Stelle steht. So darf er Briefe schreiben und Pakete von zu Hause erhalten. Ein Mithäftling klagt: „Ja, du und dein Kollege und vielleicht noch zehn von euch Polen, ihr kriegt welche, aber auch nicht alle von euch. Aber was ist mit uns Juden und Russen?" (BOROWSKI 2006:193)

Wie wird der Antisemitismus unter den Häftlingen dargestellt? Spielt er sogar eine Rolle beim Autor selbst? KLÜGER (2006:53) hört in den Geschichten über jüdische Häftlinge bei Borowski „den alten polnischen Antisemitismus“ heraus, da diese Häftlinge als besonders korrumpiert und abgestumpft dargestellt würden. SAUERLAND (2007:15) ist dagegen der Überzeugung, dass Borowski den Antisemitismus unter den Häftlingen darstelle, ohne selbst antisemitisch zu sein. Sieht man sich entsprechende Textstellen in den Erzählungen näher an, so findet man tatsächlich neben dem bereits genannten leicht weitere Belege für Borowskis kritische Haltung gegenüber dem Antisemitismus im Lager. Aber man stößt auch auf Stellen, die mindestens ambivalent sind. An einem Beispiel soll das gezeigt werden: In Bei uns in Auschwitz wird der Antisemitismus im Lager thematisiert, nachdem der IchErzähler einige leidvolle Lagererfahrungen geschildert hat:

„Das ist doch gar nichts“, sagte Witek. „Den Juden geht es noch schlechter. Bilde dir bloß nichts ein!" Sowohl über die Transporte als auch über die Juden waren die Meinungen geteilt. „Die Juden, ihr wißt, wie die Juden sind!“ meldete sich Staszek zu Wort. „Du wirst sehen, die machen mit ihrem Lager noch ein Geschäft! Sie sind sowohl im Krematorium als auch im Ghetto, und sie verkaufen die eigene Mutter für eine Schüssel Steckrüben.“ (BOROWSKI 2006:52)

Dann erzählt Staszek die tragische Geschichte eines Juden, der seinen Vater in die Gaskammer führen musste. Obwohl die Geschichte weder das Vorurteil vom Geschäftemachen bestätigt noch komisch ist, lachen die Zuhörer: „Wir lachten. Eigentlich gut, daß sie derzeit keine Arier vergasen. Alles, nur das nicht." (BOROWSKI 2006:53) Hier führt Borowski den Antisemitismus als Teil der Verrohung der Gefangenen vor. Durch den Kommentar Witeks und den Erzähler-Kommentar vor Staszeks Erzählung wird die Aufmerksamkeit des Lesers für diese Verrohung geschärft. Wenn es aber an späterer Stelle im Text nüchtern beschreibend heißt: „Doch sogleich stahlen sich einige Juden fort, um zu handeln, zu organisieren und Besuche zu machen“ (BOROWSKI 2006:71), fehlt eine erkennbare Trennung zwischen Erzähler-Ich und Intention des Textes, so dass hier das an früherer Stelle geäußerte Vorurteil vom geschäftemachenden Juden vom Text bestätigt wird. Ganz gefeit scheint der Autor Borowski nicht gegen antijüdische Stereotype gewesen zu sein, ob- 
wohl er das besondere Leiden der jüdischen Häftlinge und auch den Antisemitismus im Lager thematisiert.

Wie stellt sich die Behandlung von Borowskis Texten in den untersuchten Schulbüchern dar? Alle drei thematisieren die universalistische Deutung des Lagers bei Borowski. Tatsächlich stellt Borowski Vergleiche zwischen der Ausbeutung und der Unmenschlichkeit im Lager und der Sklaverei in der Antike an (BOROWSKI 2006:58). Auch sieht er die Demoralisierung noch nach der Schließung der Lager fortwirken, etwa in der Schlacht von Grunwald (2006:298). In allen Schulbüchern wird außerdem die Demoralisierung der Häftlinge zum Thema, dargestellt durch den ,człowiek zlagrowany (,Lagermensch '): „ein Mensch, der nur in Bezug auf das Leben im Lager denkt und entsprechend der im Lager herrschenden Moral handelt" (Barwy epok, $370)^{5}$ und drittens die Form des Werks, besonders die Erzählhaltung. Unterschiede zwischen den Schulbüchern fallen vor allem in der Art der didaktischen Vermittlung auf. Pamiętajcie gibt die Lesart der Erzählungen durch einen Kommentar vor, der vor allem auf der Monographie von TADEUSZ DREWNOWSKI (1992) basiert. Es folgen Fragen zu den Erzählungen, die mit Hilfe des Kommentars beantwortet werden können. (Pamiętajcie, 225-227). Es handelt sich also um Aufgaben mit stark paraphrasierendem Charakter. Dagegen wählt Barwy epok eine Methode, die stärker an das eigene Urteilsvermögen der Schüler und Schülerinnen appelliert. Vorgegeben sind nur biographische Informationen zum Autor und zum Werk. Schon die erste Frage enthält die Aufforderung, das Werk Borowskis in die Diskussionen zur Gestaltung der polnischen Nachkriegsliteratur einzuordnen, wobei auch die Möglichkeit in Betracht gezogen wird, dass das Werk nach einer sehr individuellen Betrachtung verlangt:

Erinnere dich an die Okkupationsdiskussionen über die Gestaltung der polnischen Nachkriegsliteratur! Mit welcher Stimme spricht Borowski? Ist es eine „,durch das Leiden beflügelte Literatur“ oder ein „Gebrülle“, oder aber lassen sich die Erzählungen von Borowski keiner dieser Kategorien zuordnen? Begründe deine Antwort! Falls du findest, dass für die Prosa von Borowski in den damaligen Diskussionen keine entsprechende Bezeichnung vorgesehen wurde, so schlage deine eigene vor!

Eine gewisse ,Leitung ' erfolgt dann allerdings noch durch die Aufforderung, bei der Beantwortung der Frage eine Aussage des Borowski-Forschers Drew-

5 Bei den Übersetzungen aus dem Polnischen war ich häufig auf die Hilfe meiner ehemaligen Kollegin Dr. Dorota Sosnicka angewiesen. Ihr sei an dieser Stelle herzlich gedankt. Für die letzte Fassung bleibe gleichwohl ich verantwortlich. 
nowski zu berücksichtigen: „Różewicz, kein Auschwitz-Häftling, ein Partisan, wurde zum Wegbereiter der Poesie nach Auschwitz. Borowski dagegen fiel die Rolle zu, die Prosa nach Auschwitz zu entdecken“ (Barwy epok, 368369). Anders als in Pamiętajcie wird auch die Rezeption Borowskis im Nachkriegspolen thematisiert, als manche Kritiker den Autor mit seinen Protagonisten identifizierten und dem Autor dann Zynismus und Nihilismus vorwarfen (Barwy epok, 368). Die Lernenden werden aufgefordert, kritische Äußerungen Borowskis über die Berichte anderer Überlebender zu überdenken, etwa Borowskis Aufforderung, weniger heroisierend und beschönigend über das eigene Verhalten zu schreiben: „Nun ja - erzählt doch endlich, wie ihr die Einweisung ins Krankenhaus gekauft habt, in gute Kommandos, wie ihr die Muselmänner in die Verbrennungsöfen geschubst habt, ... dass ein kleiner Teil des düsteren Raumes von Auschwitz auch euch gebührt..." (Barwy epok, 369). Einige der Fragen beziehen sich auf Positionen der Literaturwissenschaft oder -kritik, die in Auszügen abgedruckt sind. Hier wird allerdings nicht zu einer kritischen Reflexion eingeladen, sondern in der Regel zu einem nachvollziehenden Verstehen. So werden die Schüler aufgefordert, über den Zweck von Borowskis Schreibstil nachzudenken, dessen Stil von Andrzej Werner als „behavioristisch“ bezeichnet wird. Insgesamt sei der Sprachstil trocken und sachlich und komme dem Dokument am nächsten, obwohl er eine komplexe literarische Vision gestalte (Barwy epok, 370). Nur bei einer von insgesamt zwölf Fragen wird nahegelegt, eine eigene Position auch zu der Ansicht eines Wissenschaftlers zu beziehen: „Stimmst du der folgenden Feststellung eines Wissenschaftlers zu?" Es handelt sich um Andrzej Stanisław Kowalczyks Bemerkungen über Borowskis Selbstmord und sein Verhältnis zum „roten Totalitarismus“. Kowalczyk kommt zu dem Schluss: „Das Opfer des eigenen Lebens - in der polnischen Mythologie so hoch geschätzt - beglaubigt und bereinigt Borowski, nimmt die Bürde der Verantwortung von ihm - der Verantwortung für die Publizistik der letzten Monate seines Lebens, die in der Tat wahnhaft ist, wie von einem anderen geschrieben." (Barwy epok, 373) So begrüßenswert an dieser Stelle der Appell an eine distanzierte Lektüre ist, so kann man doch anmerken, dass ein Textbeispiel aus Borowskis Schriften aus der strittigen Zeit eine eigene Stellungnahme der Schülerinnen und Schüler erleichtern würde.

Auch das Schulbuch Między tekstami nimmt ähnlich wie Pamiętajcie eine Interpretation des Werkes vorweg, noch bevor Fragen gestellt werden. Wie problematisch eine solche vorwegnehmende Interpretation ist, soll folgendes Beispiel verdeutlichen: Es werden verschiedene Vorwürfe von Kritikern referiert, u. a. der Vorwurf, dass der Autor nicht klar zwischen Tätern und Opfern 
unterscheide. Im Schulbuch (Między tekstami, 203) heißt es dazu: „Die Antwort von Borowski auf diese Vorwürfe hinterlassen keine Zweifel. Nach seiner Ansicht ist in der ,steinernen Welt" niemand unschuldig." Obwohl sich diese Aussage auf den Autor beruft, fehlt eine Quellenangabe. Unabhängig davon, ob die Aussage tatsächlich von Borowski stammt oder eine Deutung seiner Schriften darstellt, ist sie problematisch. Man könnte entgegnen, dass die Opfer, die vom Transport direkt zu den Gaskammern geschickt wurden, gewiss keine Gelegenheit hatten, im Lager irgendeine Schuld auf sich zu laden. Das trifft vor allem auf viele jüdische Opfer zu. Obwohl die Erzählungen Borowskis für das Abitur vollständig gelesen werden müssen, druckt Między tekstami Auszüge ab und betont damit einzelne Passagen. Die gewählte Textstelle aus Bitte, die Herrschaften zum Gas wäre im Grunde sehr geeignet, die Behauptung „Niemand ist unschuldig“ aus dem Kommentar zu widerlegen. Denn es handelt sich um die letzten Seiten der Erzählung, die vom Transport aus Sosnowitz-Bendzin handelt (entspricht in der deutschsprachigen Ausgabe den Seiten 217 ab „Wieder antreten“ bis S. 221 „,brennt bereits"). Dass die auftretenden SS-Männer schuldig sind, steht außer Frage, sie gehen hier mit besonderer Brutalität vor. Über eine moralische Mitschuld der Funktionshäftlinge kann man diskutieren. Für sie ist es ein ,guter Transport“, weil Lebensmittel gegen den Hunger und Waren zum Tauschen abfallen. Aber welche Schuld haben diejenigen, die aus den Waggons kommen und noch glauben, ,dass sie schwer um ihr Überleben kämpfen werden. Sie wissen nicht, dass sie gleich sterben werden [...]“ (BOROWSKI 2006:219)? Mit der Aussage des Kommentars „niemand ist unschuldig“ wird die Perspektive dieser Todgeweihten übergangen.

Aus Bei uns in Auschwitz wird der Auszug gewählt, in dem Borowski das Konzentrationslager mit der Unterdrückung und der Ausbeutung von Sklaven in der Antike vergleicht (BOROWSKI 2006:56-58). Diese Passage lenkt die Aufmerksamkeit besonders auf die universale Zivilisationskritik Borowskis, die auch in den vergleichsweise wenigen (drei) Fragen und Aufgaben zu den Texten neben der Charakterisierung des ,Lagermenschen" eine besondere Rolle spielt: „Ist die Existenz von irgendwelchen Werten in einer solchen Welt - nach deiner Meinung - möglich? Begründe deine Meinung!““ (Między tekstami, 205) Im Schulbuch folgen anderthalb Seiten Kommentar zu Borowskis Werk aus der Monographie von Drewnowski. Dessen Schwerpunkt ist neben der Zivilisationskritik vor allem die Unterscheidung zwischen der Erzählperspektive der Ich-Erzähler und der Biographie des Autors. Zu diesem Kommentar werden fünf Verständnisfragen gestellt, die aber lediglich zur Zusammenfassung oder zur Paraphrase auffordern (Między tekstami, 206- 
207). An dieser Stelle sei bemerkt, dass in keinem der drei Schulbücher auf die Situation Borowskis und seines Ich-Erzählers als Nicht-Jude und als Funktionshäftling eingegangen wird. Es wird nahegelegt, dass die Typologie des ,Lagermenschen' auf alle Häftlinge, unabhängig von ihrem Status innerhalb der Lagerhierarchie oder Religionszugehörigkeit, ihrer Nationalität, geschweige denn von Alter oder Geschlecht, zutraf. Es wundert daher nicht, dass die Frage, ob und inwieweit die Texte Borowskis Antisemitismus im Lager thematisieren oder sogar selbst stellenweise antisemitisch gelesen werden können, nicht vorkommt.

\section{Czesław Miłosz}

Ebenfalls zum Kanon gehören heute die Gedichte von Czesław Miłosz. Als Miłosz 1980 den Nobelpreis für Literatur erhielt, lebte er seit fast dreißig Jahren im Exil, war seit zwanzig Jahren in den USA als Literaturprofessor tätig und den meisten Polen unbekannt. Erst nach der Nobelpreisverleihung durften seine Werke in Polen wieder gedruckt werden. Seit den 90er Jahren sind seine Gedichte Schullektüre. Auch seine kritische Auseinandersetzung mit dem Verhältnis der Intellektuellen zu Theorie und Praxis des Kommunismus, Verführtes Denken (Zniewolony umyst, MıOSZ 1974), wird heute in der Schule behandelt. Unter seinen Gedichten nimmt das Gedicht Campo di Fiori eine besondere Stellung ein. In allen drei Schulbüchern wird es behandelt, in Między tekstami ist es das einzige, das besprochen wird. Auch dieses Gedicht hat die Opfer zum Thema, allerdings die Opfer des Warschauer Ghettoaufstands und damit die jüdischen Opfer. Geschrieben ist es aus der Zuschauerperspektive. Und es ist diese Zuschauerperspektive, die kritisch beleuchtet wird. Geschrieben wurde das Gedicht 1943, veröffentlicht wurde es erstmals in einer jüdischen Untergrundzeitung als eines der wenigen, die von einem nichtjüdischen Autor stammten.

Miłosz vergleicht in dem Gedicht die Gleichgültigkeit der Menge während und nach der Verbrennung Giordano Brunos auf dem Campo di Fiori in Rom mit der Gleichgültigkeit der Warschauer Bevölkerung während der Niederschlagung des Ghettoaufstands. Wo heute buntes Treiben herrsche, Blumen und Früchte verkauft werden, wurde einst Giordano Bruno als Ketzer verbrannt, erinnert Miłosz in seinem Gedicht, das den Titel des auch bei Touristen bekannten Platzes trägt:

Auf diesem selben Markte

Verbrannte Giordano Bruno, 
Das Feuer, geschürt vom Henker, Wärmte die Neugier der Gaffer.

Und kaum war die Flamme erloschen,

Füllten sich gleich die Tavernen,

Körbe Oliven, Zitronen

Trugen die Händler auf Köpfen.

Ich dachte an Campo di Fiori

In Warschau an einem Abend

Im Frühling vor Karussellen

Bei Klängen lustiger Lieder.

Der Schlager dämpfte die Salven

Hinter der Mauer des Gettos,

Und Paare flogen nach oben

Weit in den heiteren Himmel.

So die zweite und dritte Strophe des insgesamt achtstrophigen Gedichts in der Übersetzung von Karl Dedecius (MitOSZ 1999:37). Milosz bezieht sich hier auf eine historische Tatsache. Tatsächlich befand sich direkt neben den Mauern des Warschauer Ghettos eine Art Rummelplatz. Das Karussell stellte seinen Betrieb auch während der Erschießung der Aufständischen nicht ein. Das lyrische Ich betont weiter, dass es ihm statt um mögliche Empörung oder um Gedanken von Vergänglichkeit vor allem um die Einsamkeit der Opfer gehe: „Ich aber dachte damals / An das Alleinsein der Opfer“. Denn das muntere Weiterleben der anderen vergrößere die Kluft zwischen den Sterbenden und der sie umgebenden Welt, so dass ihnen die Worte fehlen, zu dieser schon jetzt so weit entfernten Menge auch nur zu sprechen: ,fremd ist unsere Sprache ihnen, Als wär sie vom alten Planeten“. Tatsächlich kommt hier zu einem frühen Zeitpunkt bereits ein Bewusstsein für die sprachlichen Barrieren der Mitteilbarkeit des Schreckens zum Ausdruck (SAUERLAND 2004: 180). Wenn schließlich in der letzten Zeile das Dichterwort beschworen wird, das zum Aufruhr aufruft, ,und später nach Jahren / Auf neuem Campo di Fiori / Ein Dichterwort aufruft zum Aufruhr“, dann stellt sich das Gedicht in eine polnische romantische Literaturtradition. Und es war ja ein nationaler Aufstand in Vorbereitung, der dann im August 1944 entfesselt wurde. Man kann sich fragen, ob der Vergleich mit der Verbrennung eines Einzelnen den Massenverbrechen angemessen ist. Auch kann man nach der Position des kritischen, aber unbeteiligten lyrischen Ichs fragen. Kurz nach Campo di Fiori schrieb Miłosz ein weiteres Gedicht, das sich auf das Ghetto bezieht: 
Armer Christ schaut auf das Ghetto. ${ }^{6}$ Dieses Gedicht ist weniger leicht zugänglich und gleichzeitig beunruhigender, da es das lyrische Ich in den kritischen Blick miteinbezieht. In ihm ist von einem rätselhaften „WächterMaulwurf“ die Rede, der die Überreste der Toten berührt und zählt, vor dem sich das lyrische Ich fürchtet: „Was sage ich ihm, ich, der Jude des Neuen Testaments, / der seit zweitausend Jahren auf die Wiederkehr von Jesus wartet / Vielleicht liefert mich der zerschlagene Körper seinem Blick aus / Und er wird mich zu den Gehilfen des Todes zählen: den Unbeschnittenen." Für Jan Błoński stellen beide Gedichte, Campo di Fiori und Armer Christ schaut auf das Ghetto, Versuche dar, die moralische Mitschuld durch unterlassene Hilfeleistung als Zuschauer eines Verbrechens, das von anderen verübt wurde, zu thematisieren. Im Maulwurf aus Armer Christ schaut auf das Ghetto erkennt Błoński das schlechte Gewissen: „Das eigene moralische Gewissen verdammt (kann verdammen) den armen Christen“ (BŁOŃSKI 1996:85). Die Furcht des lyrischen Ichs im Gedicht sei dieselbe Furcht, die viele Polen bei Auseinandersetzungen über ihre Rolle während des Krieges antreibe, keinen Zweifel an ihrem Verhalten während des Krieges aufkommen zu lassen. „Die Furcht davor, unter die Handlanger des Todes gezählt zu werden. So schrecklich ist diese Furcht, diese Angst, dass wir alles tun, um sie fernzuhalten, um nicht einmal ihre Entdeckung zuzulassen“ (BŁOŃSKI 1996:86). Der Aufsatz Błońskis wurde 1987 Gegenstand der ersten öffentlich geführten Debatte in Polen über Mitverantwortung an den Verbrechen der deutschen Nationalsozialisten (BREYSACH 2005:222). Campo di Fiori blieb ein umstrittenes Gedicht. Noch im Jahr 2003 sah sich der Autor gezwungen, der zum wiederholten Male aufgetauchten These, es handle sich bei der Beschreibung der lachenden Paare auf dem Karussell nur um eine Metapher, zu widersprechen: „Dieses Gedicht habe ich geschrieben und viele Male wurde ich attackiert als Schöpfer der schädlichen Fiktion für den guten Namen der Warschauer. Es bürgerte sich ein, dass das Poem eine Metapher sei, die den Protest angesichts der Gleichgültigkeit der Stadtbevölkerung gegenüber der Tragödie im Ghetto ausdrückt.“ (MıŁOSZ 2003, zit. nach der Übersetzung von HansChristian Trepte). In Karussell beschreibt er seine Beobachtungen und seine Erschütterung, die Auslöser für sein Gedicht wurde. Ob man an einem anderen Tag, einem Werktag womöglich, ein leeres Karussell dort habe sehen

6 Ich wähle hier die Übersetzung von Karol Sauerland und somit auch seine Schreibweise des Wortes Ghetto, dessen Neuübersetzung von Biedny chrześcijanin patrzy na getto (SAUERLAND 2004:180-181) überzeugender ist als die verbreitete von Karl Dedecius: Armer Christ sieht das Getto (MILosz 1999:61). 
können, sei für den Wirklichkeitsgehalt und für die Wahrheit dieses einen Gedichts unerheblich (MıOsz 2003). Ryszard Matuszewski hatte in einem Artikel in Rzeczpospolita (10./11.5. 2003) berichtet, er habe bei seinen Straßenbahnfahrten, die ihn auch zu jener Zeit am Karussell vorbeiführten, stets ein unbewegtes Karussell gesehen. Daraus schloss er, es müsse sich bei Miłosz um eine metaphorische Darstellung handeln. Dieser Artikel wurde der aktuelle Anlass für den Widerspruch von Miłosz.

An dieser Stelle sei an eine ähnliche Auseinandersetzung zwischen dem Autor Paul Celan und seinen Interpreten über das kanonische Gedicht Todesfuge erinnert. Celan bestand immer wieder auf der sehr wirklichkeitsnahen Bedeutung seiner Wortwahl, um allzu metaphorischen Deutungen zu widersprechen. ${ }^{7}$ Eine weitere bemerkenswerte Ähnlichkeit dieser beiden kanonischen Gedichte besteht im Auseinanderfallen von Autor-Intention und Rezeption. Celan war die Neigung mancher Deutscher, das Gedicht entgegen des anklagenden Grundtones, „Der Tod ist ein Meister aus Deutschland“, als Versöhnungsgeste zwischen Juden und Deutschen zu lesen, so unheimlich oder sagen wir besser zuwider, dass er es in Lesebüchern am liebsten nicht mehr abgedruckt gesehen hätte. Miłosz' Gedicht Campo di Fiori wurde wiederholt als Anklage verstanden und der Autor als Nestbeschmutzer, wohingegen der Autor betonte, gar keine Anklage im Sinn gehabt zu haben.

Wenden wir uns nun der Behandlung des Gedichts in den Schulbüchern zu. Pamiętajcie gibt wie schon bei Borowski eine allgemeine Deutung vor, deren Schwerpunkt auf der Moralität der Miłosz'schen Poesie im Allgemeinen und auf der Thematisierung von menschlicher Gleichgültigkeit in diesem besonderen Fall liegt (Pamiętajcie, 248). Die einzige (allerdings zweiteilige) Aufgabe fordert auf: „Beschreibe den symbolischen Sinn der Bilder, die im Gedicht Campo di Fiori von Czesław Miłosz enthalten sind“ und bittet um eine Deutung der letzten Zeile des Gedichts (Pamiętajcie, 250). Die Fragestellung legt durchaus die von Miłosz selbst kritisierte Lesart des Karussells als Fiktion oder Metapher nahe, da lediglich nach „Bildern“ und deren „symbolischem Gehalt" gefragt wird. In Barwy epok folgen auf biographische Informationen zum Dichter und das Gedicht selbst mehrere Fragen zur Diskussion, wozu auch eine Frage zum Realitätsgehalt der dritten und vierten Strophe gehört: „Denke darüber nach, ob und auf welche Weise die dritte und

7 In einem Brief an Walter Jens vom 19.5.1961 schrieb er: „Das ,Grab in der Luft“ - lieber Walter Jens, das ist, in diesem Gedicht, weiß Gott weder Entlehnung noch Metapher.“ (CELAN 2003:608) 
vierte Strophe illustriert werden könnten, damit ihre Aussage und Atmosphäre wiedergegeben werden könnten! Wärst du für eine realistische Darstellung oder eher für ein Symbol, eine Metapher? Begründe deine Wahl - beschreibe die vorgeschlagene Komposition!“ (Barwy epok, 314). Hier wird eine Diskussion angestoßen, bemerkenswerterweise in Verbindung mit einer kreativen Aufgabe. Man könnte lediglich einwenden, dass die Lernenden letztlich über Realismus oder Metaphorik des Gedichts ohne Informationen über die reale Situation nicht urteilen können. Das dritte Schulbuch, Między tekstami, dokumentiert mit Textauszügen die jüngste Debatte über den Wirklichkeitsgehalt von Campo di Fiori. Abgedruckt sind Auszüge der Beiträge von Miłosz und Matuszewski sowie Kommentare von Tomasz Szarota und Aleksander Kopiński. Eine Darstellung von Marek Edelman aus HANNA KRALLs Buch Dem Herrgott zuvorkommen (1992) ergänzt die Debatte. Edelman erinnert sich an das Karussell aus seiner Sicht, d.h. von der anderen Seite der Mauer: Auf die Frage „Konnte man über die Mauer hinweg auf die arische Seite schauen?" antwortet er:

Ja. Die Mauer reichte nur bis zum ersten Stock. Vom zweiten sah man schon die Straße drüben. Wir sahen ein Karussell und Leute, wir hörten die Musik und hatten entsetzliche Angst, daß diese Klänge uns übertönen und diese Leute uns nicht bemerken würden, daß überhaupt niemand auf der Welt aufmerksam würde - auf uns, diesen Kampf und die Toten... Wir hatten Angst, diese Mauer könne so hoch sein, daß nichts, keine Nachricht von uns, hinüberdrang. (KRALL 1992:13f.)

Außerdem ist ein Foto abgedruckt, das das Karussell am Rande der Ghettomauer zeigt. Da die gewählten Auszüge unterschiedlich in ihren Positionen und Argumentationen sind, dürfte die Grundlage für eine differenzierte und lebhafte Auseinandersetzung über das Gedicht gelegt sein. In den Aufgaben werden die Lernenden aufgefordert, die jeweiligen Sichtweisen zu überdenken und begründet Stellung zu beziehen. Außerdem sollen sie sich besonders zu einigen Sätzen von Miłosz äußern: „Es ist ein Beispiel, wie gefährlich Treue gegenüber den Tatsachen ist, weil die Tatsache allein bisweilen aussieht wie ein Fantasieprodukt und dank dem an gewaltiger Reichweite gewinnt.“ (Mı૦OSZ 2003) Es ist tatsächlich das einzige Mal, dass bei den untersuchten Darstellungen in den drei Schulbüchern gegensätzliche Positionen gegenübergestellt werden, die dann von den Schülern beurteilt werden sollen. 
Marita Meyer

\section{Hanna Krall}

Beim dritten kanonischen Werk handelt es sich um Dem Herrgott zuvorkommen von Hanna Krall. Dem Buch liegen Gespräche mit Marek Edelman, dem einzigen überlebenden Anführer des Warschauer Ghettoaufstands von 1943, zugrunde. Das zugrunde liegende Interview ist noch in der dialogischen Struktur des Textes präsent. Passagenweise wird die Darstellung von der Autorin in den Erzählmodus überführt, wobei die Interviewerin auch die Perspektive des Befragten übernimmt. Mit Hilfe der dialogischen Struktur können Zweifelsfälle thematisiert werden, Nachfragen gestellt werden sowie die Möglichkeiten und die Grenzen des Sprechens und Schreibens diskutiert werden. Diese Reportage befasst sich nicht nur mit dem Ghettoaufstand in der Erinnerung von Marek Edelman, sondern auch mit der Gegenwart des Befragten, vor allem seinem beruflichen Alltag als Herzchirurg. Diese beiden Ebenen werden durchaus kunstvoll aufeinander bezogen und mit der dritten Ebene, den Reflexionen über die Erzählweise und den Ablauf der Ereignisse, verwoben, so dass man mit guten Gründen von einer literarischen Reportage sprechen kann. Das Buch erschien 1977, also fünfunddreißig Jahre nach dem Aufstand. Nicht nur der Aufstand selbst bildet das Thema des Buches, sondern vor allem die Art der Erinnerung an diesen Aufstand, wobei die Unterschiede zwischen der offiziellen Erinnerung und der persönlichen Erinnerung von Marek Edelman eine Art Leitfrage ist. Dies betont bereits der erste Teil des Buches, eine Art Prolog, in dem erklärt wird, warum Marek Edelman dreißig Jahre lang geschwiegen hat. Als er kurz nach dem Krieg über den Aufstand redete, redete er nicht so, wie man es von ihm erwartete:

Wie sich zeigte, hatte er nicht gesprochen, wie es sich gehört hätte. „Und wie hätte es sich gehört?" fragte er. Voller Haß und Pathos, schreiend - nur der Schrei allein ist imstande, all das auszudrücken. Also taugte er von Anfang an nicht zum Redner, denn er konnte nicht schreien. Und er taugte auch nicht zum Helden, denn in ihm war kein Pathos. (KRALL 1992:23)

Auch noch dreißig Jahre später, als die Interviewerin ihn zum Reden bringt, löst eine Vorveröffentlichung Empörung aus. In ihr hatte Marek Edelman u.a. über Anielewicz, den Anführer des Aufstands, erzählt, er habe vor dem Krieg mit seiner Mutter Fische mit roter Farbe bestrichen, um sie frisch aussehen zu lassen (KRALL 1992:12). Viele Leser, auch solche aus dem Ausland, in deren Sprachen das Interview übersetzt worden war, fanden, ,dass er alles seiner Größe entkleidet habe“ (KRALL 1992:18). Kralls Gespräche mit Edelman über die Erinnerungen an den Aufstand und seine Bedeutung fordern zum Nachdenken über die offizielle Erinnerungspolitik heraus. Mit 
Aleida Assmann kann man sagen, Krall versucht gemeinsam mit Edelman, die erstarrte Erinnerung wieder zu einer fließenden Erinnerung zu machen. ${ }^{8}$ In dieser fließenden oder lebendigen Erinnerung kann es durchaus Zweifel am Ablauf der Ereignisse geben. Gab es die israelische Flagge neben der polnischen Flagge im Ghetto während des Aufstands? Nach dem Krieg erinnern sich viele an die Flaggen. Edelman aber hat sie nicht gesehen, hält dies für undenkbar, da es gar keinen Stoff für Flaggen gegeben habe (KRALL 1992:128). Ein weiteres Beispiel ist die Uneinigkeit der Überlebenden über die Zahl der Aufständischen. Edelman tendiert zu einer geringeren Zahl und hält größere Zahlen für Wunschdenken (KRALL 1992:21-22). Es gehört nicht zur Intention des Buches, diese faktischen Zweifelsfälle zu klären. Aber es wird deutlich, dass die Fakten eng mit den Deutungen des Geschehens zusammenhängen. Es werden Konflikte lebendig, etwa wenn es um die Frage der Widerstandsbewegung ging, wessen Leben gerettet werden soll. Und nicht ausgespart wird die Beschreibung der Begegnung mit den Nachfahren derjenigen, deren Tod beschlossen wurde: „Hattet ihr überhaupt ein Recht, für ihn den Tod zu wählen?“ (KRALL 1992:75) Die Interviewerin reagiert zuweilen auch mit Unverständnis und Sprachlosigkeit (KraLL 1992:16), sie stellt auch unbequeme Fragen, nach unterlassener Hilfeleistung etwa (KRALL 1992:55), und sie reflektiert gemeinsam mit Edelman über die Qualität der Geschichten, auch über die „kitschigen Storys“ (KRALL 1992:51). Dass diese Dekonstruktion der heroischen Version des Aufstands nachhaltig gewesen ist, hat 25 Jahre später der Politologe DAN DINER (2000:20) bestätigt:

Seit Hanna Krall dem verstummten Marek Edelman Sprache und Stimme wiedergab, hat sich alles weitere Reden und Schreiben über den Aufstand gewandelt. Jedenfalls ist nach diesem Bericht aus so manch unerbetenem heroisierenden Nach-Erzählen des Geschehens die sonsthin übliche falsche Emphase gewichen. Statt dessen ist ein eher betretenes Schweigen eingekehrt.

Ob mit dieser Dokumentation des Zweifelns auch gleichzeitig die Autorität der Zeugenschaft selbst in Frage gestellt wird, wie BREYSACH (2005:375) meint? Gewiss stellen Edelman und Krall der dekonstruierten Geschichte vom heldenhaften Aufstand keine andere Geschichte des Aufstands entgegen, zu groß ist das Misstrauen in große und ungebrochene Erzählungen, eine Haltung, in der man auch postmoderne Züge erkennen kann. Aber statt der einen

8 Im Anschluss an Georg Simmel und andere Kultursoziologen hat AssmanN (1991:182) die metaphorischen Begriffsbestimmungen von „fest“ und „flüssig“ verfolgt und kommt zu dem Ergebnis, dass kulturelle Tätigkeit „Oszillieren zwischen Verfestigung und Verflüssigung" bedeutet. 
Geschichte werden doch einzelne Geschichten von Menschen erzählt, deren mutiges oder humanes Verhalten in der großen Erzählung nicht vorkommt. Tzvetan Todorov hat in seinem Nachwort zur deutschen Ausgabe von „Alltagstugenden“ gesprochen, die Edelman den heroischen Tugenden gegenüberstelle. Dazu gehöre, sich nicht demütigen zu lassen sowie die „Sorge um andere“ (KRALL 1992:163). Den Selbstmord von Czerniaków verurteilt Edelman als sinnlos, aber der junge Bräutigam, der mit seinem Körper die Braut vor dem auf sie gerichteten Gewehr schützt und dabei eine Hand verliert, gilt ihm als vorbildlich: „Darum eben ging es: daß einer da war, der deinen Leib notfalls mit der eigenen Hand beschirmte“ (KRALL 1992:60). Für die Vermittlung dieser persönlichen Einsichten legt Edelman durchaus seine Autorität als Augenzeuge in die Waagschale. Eine weitere nicht gering zu achtende Leistung liegt im Gespräch selbst: Als Leser werden wir Zeugen einer Übereignung von Erinnerung von einem Augenzeugen an eine interessierte Zuhörerin, die mit ihrer Nähe zum Zeugen und durch ihre Bereitschaft zu Verständnis und Durchdringung des Geschehens selbst zu einer Zeugin wird. Nur so ist sie in der Lage, das ,verdichtete Zwiegespräch“, wie Diner es nennt, zu verfassen. Die Interviewerin, die zur Erzählerin wird, hat sich die Geschichten von Edelman angeeignet, fragend, auch widersprechend, aber wohlwollend. Vielleicht kann man hier mit GEOFFREY HARTMAN (2007:73) von ,intellektueller Zeugenschaft“ sprechen. Die Lakonie und die Distanz zu jeder Art von Pathos, die stilbildend in allen weiteren Werken der Autorin werden und als Merkmal stets hervorgehoben werden ${ }^{9}$, zeichnen bereits das Gespräch zwischen Edelman und Krall aus.

In den Schulbüchern sind Krall und Dem Herrgott zuvorkommen nur auf jeweils wenigen Seiten präsent, was nicht darüber hinwegtäuschen sollte, dass das gesamte Buch Pflichtlektüre ist. Die Behandlung in den Schulbüchern erlaubt nur einen Einblick in die Schwerpunktsetzung. Das galt auch für die bisher besprochenen Werke, für dieses gilt es in besonderem Maße. Pamiętajcie widmet dem Thema insgesamt eine Seite, die aus biographischen Informationen über Krall und Edelman sowie einer halben Seite Kommentar zu Dem Herrgott zuvorkommen besteht. Dieser Kommentar liefert eine knappe Einordnung des Werkes in die ,literatura faktu', die Dokumentarliteratur, und in die Tradition der Reportage. Wie bei den Werken von Borowski und Miłosz gibt der Kommentar eine Deutung vor, die in diesem Fall eine recht

9 So auch von IRMELA vON DER LÜHE (1999:108), die die Rezeption von Hanna Krall in Deutschland untersucht hat. 
allgemeine ist. Hervorgehoben wird der Zeugnischarakter des Buches, die moralische Dimension von Edelman sowie die antiheroische Haltung von Buch wie Protagonisten. Die einzige Aufgabenstellung zum Werk enthält dann eine Aufforderung, sich mit der Komposition auseinanderzusetzen: „Die Reportage kennt keine Fiktion. Besprich die Komposition des Werks!“ (Pamiętajcie, 244)

Auch Barwy epok behandelt Kralls Werk unter dem Oberthema der Dokumentarliteratur neben Kazimierz Moczarski und Ryszard Kapuściński. In diesem Schulbuch wird keine Deutung vorgegeben. Nach einer knappen Information zur Autorin folgen zwei Textauszüge, zu denen vier Fragen gestellt werden. Der erste gewählte Auszug ist geeignet, den Titel des Buches zu erklären, da er eine Beziehung zwischen Edelmans Tätigkeit im Lager und seinem Beruf als Herzchirurg herstellt. Hier wie dort hat Edelman mit Todgeweihten zu tun: „Meine Aufgabe bestand darin, möglichst viele von ihnen zu retten, und einmal - dort unter dieser Palme - kam ich darauf, daß es keine andere Aufgabe war als jene vom Umschlagplatz. Da hatte ich auch am Tor gestanden und Leute aus der Schar der Verurteilten herausgeholt.“ (KRALL 1992:102) Im zweiten Auszug berichtet Edelman von der Niederschlagung des Aufstands, die auch darin besteht, dass die Deutschen Teile des Ghettos in Brand stecken. Der Ausschnitt beschreibt den sich ausbreitenden Brand und seine Opfer: Sie verbrennen beim Versuch, durch die Flammen zu fliehen oder beim Versuch, durch unterirdische Gänge zu entkommen. Diese Beschreibung ist im Werk in kritische Reflexionen Edelmans über die Konventionen des Erzählens eingebettet. Dort beginnt Edelman zunächst mit dem Bericht von der Brandstiftung aus der Sicht eines hereinstürzenden ,jungen Burschen“, korrigiert sich dann aber, ,das ist kein seriöser historischer Bericht“, und es gehe auch ,alles zu sehr ins Detail“ (KRALL 1992:109). Er beginnt von neuem zu erzählen, diesmal aus der Perspektive der Täter: „Die Deutschen fangen also an, das Ghetto in Brand zu stecken.“ So beginnt auch der für das Schulbuch gewählte Auszug. Der Auszug endet mit dem resignierten Fazit Edelmans: ,Ja, das war der chronologische Ablauf der Ereignisse. Der historische Ablauf ist, wie sich zeigt, nicht mehr als eine Reihenfolge des Sterbens.“ (KraLl 1992:111) Die Skepsis Edelmans gegenüber der konventionellen Art des historischen Erzählens wird hier bestenfalls angedeutet. Abgeschnitten ist der folgende Absatz aus Dem Herrgott zuvorkommen, der noch in engem Zusammenhang mit dem gewählten Auszug steht und in dem die fehlende Erinnerung an das individuelle Schicksal beklagt wird: „Aber wer weiß etwas von dem jungen Menschen, der verschüttet werden mußte, weil Rauchschwaden in den Keller drangen? Wer 
weiß heute etwas von diesem Jungen?“ (Krall 1992:111) Erst in diesem Zusammenhang wird die Kritik Edelmans an einer Geschichtsschreibung deutlich, die sich auf die Ereignisgeschichte und damit häufig auf die Tätergeschichte konzentriert und deren auf Sachlichkeit und Abstraktion ausgerichtete Darstellungsweise das individuelle Schicksal vernachlässigt.

Dem Werk Kralls wird im Schulbuch Barwy epok eine größere Bedeutung beigemessen als im Schulbuch Pamiętajcie, aber es werden weder die antiheroische Erzählweise noch die Infragestellung konventioneller Geschichtsschreibung thematisiert. Auch keine der vier Fragen weist explizit in diese Richtung. Die Fragen thematisieren den Unterschied von Geschichte und Erinnerung im Allgemeinen, die erzählerischen Mittel der Darstellung, das Sprechen über den Tod sowie die Rolle Edelmans während des Aufstands. Die vierte und letzte Frage zitiert eine Aussage Kralls aus einem Interview mit Katarzyna Bielas (Gazeta Wyborcza, 13.6.1997, zit. nach Barwy epok, 416) mit der Aufforderung, zur Aussage entsprechende Passagen im Werk zu finden. Krall: „Ich muss Dinge hören, die mich dazu bringen zu fragen: Warum? Ich muss auch den Schatten einer Chance sehen, dass die Antwort irgendetwas Allgemeines enthält, dass ich irgendetwas Neues über ein paar uralte Angelegenheiten erfahre: über die Liebe, den Tod, die Angst, die Ehre, den Mut..."

Das dritte Schulbuch, Między tekstami, bietet ebenfalls Auszüge aus Dem Herrgott zuvorkommen und Fragen dazu an. Mit der Entscheidung für den ersten Textauszug wird bereits Aufmerksamkeit für die kritische Haltung des Werks gegenüber festgefahrenen Sichtweisen auf den Aufstand geschaffen. Hier wird von den Überlegungen der Aufständischen über die Art des Vorgehens berichtet. Sie wissen, dass ihre Entscheidungsfreiheit nur soweit reicht, die Art des Sterbens frei zu wählen. In diesem Kontext taucht auch der häufig zitierte Satz „Die Menschheit hat sich ja darauf geeinigt, daß das Sterben mit der Waffe schöner ist als das ohne Waffen“ (KRALL 1992:17) auf. Interessanterweise wurde auch für dieses Schulbuch als zweite Textstelle diejenige über das brennende Ghetto gewählt. Allerdings werden in Między tekstami anders als in Barwy epok auch die kritischen Vor- und Nachbemerkungen zur Schilderung der Ereignisse zitiert.

Die beiden Fragen und Aufgaben orientieren sich eng an den Textauszügen. Die erste ist bezogen auf die erste Textstelle und lautet: „Erkläre, warum für Hanna Kralls Gesprächspartner die Art des Sterbens wichtiger als der Tod selbst war." Die zweite schließt an die Überlegungen Edelmans aus dem zweiten Auszug an und fordert dazu auf, den Unterschied zwischen Augen- 
zeugenbericht und historischer Darstellung, den Marek Edelman anspricht, am Text selbst deutlich zu machen (Między tekstami, 191). Insgesamt stellt sich allein die Behandlung im dritten untersuchten Schulbuch, Między tekstami, so dar, dass man den Eindruck gewinnt, dass die vergangenheitspolitische Bedeutung des Werks thematisiert wird. Ob die Komplexität und die Besonderheit der Erzählform von Krall behandelt wird, lässt sich an diesen Fragen zwar nicht erkennen. Aber zumindest sind die Einbettung in das Oberthema ,świadkowie'(,Zeugen') zusammen mit so unterschiedlichen Werken wie Moczarskis Erinnerungen und den Gedichten von Miłosz Hinweise darauf, dass die Grenzen zwischen Dokument und Literatur offen gehalten werden. Auf diese Problematik zielt auch eine Stellungnahme des Historikers und Essayisten Jerzy Jedlicki im das Thema einleitenden Teil, die den Titel trägt: „Sprechen die Fakten für sich?“ Jedlicki zeigt an einem Beispiel, dass Fakten Beurteilungen und Vorverständnissen unterliegen. Und dass schon die Wortwahl in ein spezifisches Schema von Deutungen führe:

Man kann sagen „Die Deutschen haben die Juden ermordet“. Man kann sagen: „Die Nazis haben die Juden ermordet“. Man kann sagen: „Die Juden ließen sich ohne Widerstand ermorden“. Man kann sagen: „Die Henker verloren jegliche Art von Menschlichkeit“. Und man kann sagen: „Menschen haben Menschen dieses angetan.“ (Między tekstami, 190)

\section{Schlussfolgerungen}

Vergleicht man den polnischen expliziten Lektürekanon mit dem deutschen impliziten Lektürekanon, so muss zunächst festgehalten werden, dass es sich bei den polnischen Lektüren sämtlich um Werke handelt, deren literarische Qualität unbestritten ist. Auch gibt es keine Kluft zwischen Literaturwissenschaft und Schuldidaktik. Die in der Schule behandelten Werke gehören auch zum Kanon der Literaturwissenschaft. Dass auf der polnischen Seite ausnahmslos formal und inhaltlich anspruchsvolle und herausfordernde Werke auf dem Lehrplan stehen, während auf der deutschen Seite eine Tendenz zur Schreckensvermeidung oder zur entlastenden Lektüre zu erkennen ist, hat sicher auch mit der Entscheidung zu tun, das Thema in Polen erst im letzten Jahr vor dem Abitur zu behandeln. In Deutschland führt die frühe Thematisierung zur Wahl von ,zumutbaren' Lektüren und wohl auch manchmal zu Überdruss, bevor komplexere Werke behandelt werden können. Ganz von der Hand zu weisen ist aber der Eindruck nicht, dass erklärte gute Absichten von - vielleicht unbewussten - Wünschen nach Verdrängung unterlaufen 
werden. Besonders die weitverbreitete Lektüre des Vorlesers mit der Figur einer Täterin, die mitleiderregend wirkt, legt nahe, Zusammenhänge zu den gesamtgesellschaftlichen Tendenzen $\mathrm{zu}$ sehen, wie sie der Zeithistoriker NORBEIT FREI (2005:14) beschreibt: Mag auch der radikale Perspektivenwechsel von den Opfern der Deutschen zu den Deutschen als Opfern noch immer die Ausnahme sein, so gibt es doch Tendenzen einer ,privatistischen Geschichtsbetrachtung, in der sich die Unterschiede zwischen Tätern, Opfern und Mitläufern verwischen“. Es mehren sich Hinweise, dass die zweite Generation sich mit der Elterngeneration auszusöhnen versucht und an die dritte Generation ein geschöntes Bild der Vergangenheit weitergibt. Der Soziologe HARALD WELZER (2003:54) hat dies vor allem in Untersuchungen zum Familiengedächtnis nachgewiesen. Etwa zwei Drittel aller Geschichten, die Kinder und Enkel über Angehörige während der NS-Zeit erzählen, handeln davon, dass ihre Eltern bzw. Großeltern Opfer der NS-Vergangenheit und/ oder Helden des alltäglichen Widerstands waren. Dabei nimmt die Neigung, Opfer- oder Widerstandsgeschichten zu erzählen, mit dem Maß des Wissens über die NS-Zeit nicht etwa ab, sondern zu. Offensichtlich besteht der starke Wunsch der Nachfahren, keine Verbindung zwischen den grausamen Tatsachen und den als liebenwert empfundenen Familienangehörigen herzustellen. „Je fundierter das Geschichtswissen ist, desto größer wird die subjektiv empfundene Notwendigkeit, die eigene Familie vor diesem Wissen zu schützen - das heißt, sie aus dem historischen Zusammenhang herauszunehmen, über den man so gut Bescheid weiß“ (WELZER 2003:78). Der Holocaust existiert in diesen Familiengesprächen nur auf Nachfrage: ,er hat seinen Ort in dem kognitiven Universum dessen, was man über Geschichte weiß, nicht im Familiengedächtnis.“ (WELZER 2003:210) Der Erfolg des Vorlesers könnte darin bestehen, dass einerseits eben diese gern verdrängte Verbindung von Holocaust und nahestehenden Menschen im Roman hergestellt wird, andererseits aber im fiktiven Raum des Romans weitreichende (bis unrealistische) Möglichkeiten der moralischen Entlastung aufgezeigt werden. Sollte diese Hypothese zutreffen, die durch eingehendere Rezeptionsforschung überprüft werden müsste, dann würden sich Familiengedächtnis und offizielles Gedächtnis in der schulischen Diskussion begegnen. Die Notwendigkeit einer kritischen Behandlung des Romans würde damit allerdings noch zunehmen.

Als Alternative, besonders wenn es um das berechtigte Interesse an den Tätern geht, wäre nochmals auf Die Ermittlung von Peter Weiss zu verweisen. Das Dokumentardrama über den Frankfurter Auschwitzprozess stellt als eines der wenigen Werke Täter- und Opferperspektive gegenüber. Mit seinem universalistischen Ansatz ist der Autor Peter Weiss dem Autor Tadeusz Bo- 
rowski nahe. Auch deutsche Schülerinnen und Schüler könnten, würde das Drama häufiger gelesen, sowohl etwas über die „steinerne Welt“ des Lagers erfahren als auch über die anhaltende Verhärtung der Täter in der Nachkriegszeit.

Weiter fällt auf, dass der Verzicht auf einen festgelegten Kanon in Deutschland nicht unbedingt zu mehr Beweglichkeit und Aktualität in den Lektürelisten führt. Von dem Vorleser abgesehen stehen die deutschen Lektüren bereits seit Jahrzehnten auf dem Stundenplan. In Polen dagegen spiegeln sich im Kanon deutlich die politischen Veränderungen der letzten Jahrzehnte. Mit Kralls Werk über den Ghettoaufstand ist außerdem ein Buch im Kanon vertreten, das ritualisierte Formen des Gedenkens thematisiert und die Bedeutung der Vergangenheit für die Gegenwart reflektiert. Eine ähnliche Funktion könnte Klügers weiter leben im deutschen Schulunterricht erfüllen, es hat sich aber (vielleicht noch) nicht als Schullektüre durchgesetzt. In den tatsächlich praktizierten Kanons nehmen allein die Gedichte Todesfuge und Campo di Fiori eine vergleichbare Stellung ein. Auch wenn ästhetisch sicher Unterschiede zwischen den Autoren Celan und Miłosz bestehen, so handelt es sich doch in beiden Fällen um zentrale Werke der Holocaustliteratur. Beide Gedichte erinnern an die Verbrechen und beide fragen nach der Rolle der Kunst bzw. des Künstlers. Beide Gedichte wirkten provozierend auf ihr Publikum und beide avancierten dennoch zur Schullektüre. Beide Gedichte verlangen nach Kontextualisierung, soll ihre provozierende Wirkung lebendig bleiben.

Betrachtet man die polnischen Schullektüren als Teil des kulturellen Gedächtnisses, dann fällt auf, dass sie keineswegs ausschließlich auf die Opferrolle fixiert sind. Zwar gibt es in Polen eine starke Tradition der Opferwahrnehmung, Stichwort ,Christus der Völker', die fraglos durch die Verbrechen an der polnischen Bevölkerung im 20. Jhd. leidvoll aufs Neue mit Realität aufgefüllt und wiederbelebt wurde. Diese Tradition spielt denn auch im nationalen Gedenken Polens keine geringe Rolle. ${ }^{10}$ Die Schullektüren aber, die als kanonisierte Lektüren durchaus Teil des offiziellen nationalen Gedächtnisses sind, zeigen ein differenzierteres Bild. Wenn die drei kanonischen Werke über die Massenverbrechen etwas eint, dann ist es gerade

10 Assmann (2006:262) hat Polen als Beispiel für ein europäisches Land gewählt, „wo sich der nationale Mythos weiterhin um die Opferrolle dreht. [...] Im Lichte dieses tief eingeschliffenen Musters kultureller Erfahrungsverarbeitung ist es nahezu unmöglich, den Status anderer - der jüdischen - Opfer anzuerkennen und sich auch mit eigener Schuld - etwa der Geschichte des katholischen Antisemitismus - auseinanderzusetzen." 
die Infragestellung einer sakrosankten Opferrolle. Zentrale Bedeutung besitzt die Perspektive des Zuschauers und des Augenzeugen, eine Perspektive, die in der gesamten polnischen Literatur über den Holocaust eine wichtige Rolle spielt (BREYSACH 2005:397). Auch kann man nicht behaupten, dass die jüdischen Opfer übergangen würden, wenn auch der konkurrierende Status der Opfergruppen manchmal den Blick trüben kann. Dieser für Polen insgesamt beeindruckende Befund wird allerdings durch die Schulbücher relativiert. Die Fragestellungen und die Begleittexte der Schulbücher werden der Komplexität der genannten Werke nicht immer gerecht. Vor allem das kritische und selbstkritische Potential der literarischen Werke wird häufig nicht zum Thema gemacht. So wird zwar ausführlich die Darstellung des sogenannten ,Lagermenschen “ und seine Demoralisierung thematisiert, aber nicht die Hierarchie der Opfer, wie sie Borowski beschrieben hat, wozu auch die besonders schlechte Situation der jüdischen Opfer gehört. Bei der Behandlung von Campo di Fiori werden konkrete Fragestellungen vermieden, was den Eindruck erweckt, dass die provozierende Dimension des Gedichts abgeschwächt werden soll. Eine Ausnahme bildet hier das Schulbuch Między tekstami. Dasselbe Schulbuch ist ebenfalls das einzige unter den drei untersuchten Schulbüchern, das die kritischen Passagen zur Geschichtsschreibung und zur Vergangenheitspolitik aus Dem Herrgott zuvorkommen in den Mittelpunkt der Diskussion rückt.

Man möchte den deutschen Lehrenden mehr Mut zu herausfordernder Lektüre wünschen und den polnischen Lehrenden mehr Vertrauen in die Urteilsfähigkeit der Lernenden. Bei einem denkbaren Austausch von Lektüren - was mit Blick auf ein europäisches Gedächtnis naheliegt -, würde die deutsche Seite vielleicht stärker profitieren als die polnische. Aber ähnlich wie im Geschichtsunterricht könnte - auf beiden Seiten - die gegenseitige Kenntnisnahme bereits zu einer Erweiterung des Blickfeldes und auch zu einer differenzierteren Fremd- und Selbstwahrnehmung führen.

\section{Literatur}

Assmann, Aleida (1991): Fest und Flüssig: Anmerkungen zu einer Denkfigur. In: Assmann, Aleida / Harth, Dietrich (eds.): Kultur als Lebenswelt und Monument. Frankfurt (M.), 181-199.

- (2006): Der lange Schatten der Vergangenheit. Erinnerungskultur und Geschichtspolitik. München.

BECKER, JUREK (1969 / 1982): Jakob der Lügner. Frankfurt (M.). 
BŁońSKI, Jan (1996): Die armen Polen blicken aufs Getto. Aus dem Polnischen von Karin Wolff. In: Klecel, Marek (ed.): Polen zwischen Ost und West. Polnische Essays des 20. Jahrhunderts. Frankfurt (M.), 76-93.

BobińSki, Witold / Janus-SitarZ, AnNa / KoŁCZ, BoguSŁaw (2004): Barwy epok. Kultura i Literatura 3. [Farben der Epochen. Kultur und Literatur 3]. Warszawa.

BÖHLER, JOCHEN (2006): Auftakt zum Vernichtungskrieg. Die Wehrmacht in Polen 1939. Frankfurt (M.).

Borodziej, WŁodzimierz (2005): Polen während des Zweiten Weltkrieges in deutschen Schulbüchern. In: KERSKI / OWCZAREK, 87-94.

Borowski, TADEUSZ (2006): Bei uns in Auschwitz. Aus dem Polnischen von Friedrich Griese. Frankfurt (M.).

Braese, StePhan et al. (eds.) (1998): Deutsche Nachkriegsliteratur und der Holocaust. Frankfurt (M.)/New York.

Braese, StePhan (ed.) (1998a): In der Sprache der Täter. Neue Lektüren deutschsprachiger Nachkriegs- und Gegenwartsliteratur. Opladen.

BREYSACH, BARBARA (2005): Schauplatz und Gedächtnisraum Polen. Die Vernichtung der Juden in der deutschen und polnischen Literatur. Göttingen.

Celan, Paul (2003): Die Gedichte. Kommentierte Gesamtausgabe in einem Band. Hrsg. und kommentiert von Barbara Wiedemann. Frankfurt (M.).

DINER, DAN (2000): Laudatio auf Hanna Krall. Leipziger Buchpreis zur Europäischen Verständigung. Frankfurt (M.), 19-27.

DREWNOWSKI, TADEUSZ (1992): Ucieczka z kamiennego świata. [Ausbruch aus der steinernen Welt]. Warszawa.

Eke, Otto / Steinecke, Hartmut (eds.) (2006): Shoah in der deutschsprachigen Literatur. Berlin.

Feuchert, Sascha / Hofmann, Lars (2005): Bernhard Schlink. Der Vorleser. Lektüreschlüssel für Schüler. Stuttgart.

FINGERHUT, KARLHEINZ (1994): Stellungnahme zum Kanon-Problem für die Lehrplankommission Gymnasium SI NRW. In: KULTUSMINISTERIUM NRW: Lektüre von Ganzschriften im Fach Deutsch der Sekundarstufe 1 des Gymnasiums in NRW. Frechen, 51-53.

FreI, NORBERT (2005): 1945 und wir. Das Dritte Reich im Bewußtsein der Deutschen. München.

HaHN, HANS-JOACHIM (2005): Repräsentationen des Holocaust. Zur westdeutschen Erinnerungskultur. Berlin.

Hartman, GeOFFrey (2007): Die Ethik des Zeugnisses. Ein Interview mit Geoffrey Hartman. In: ElM, MichAEL / KÖSSLER, GOTTFRIED (eds.): Zeugenschaft des Holocaust. Zwischen Trauma, Tradierung und Ermittlung. Frankfurt (M.), 52-76.

HofmanN, MichaEL (2003): Literaturgeschichte der Shoah. Münster. 
Marita Meyer

Kerski, BASIL / OWCZAREK, ZDZISŁAW (eds.) (2005): Ist gemeinsame Erinnerung möglich? Czy możliwa jest wspólna pamięć? Berlin.

KeRTÉSZ, IMre (1996): Roman eines Schicksallosen. Aus dem Ungarischen von Christina Viragh. Berlin.

- (2003): Die exilierte Sprache. Essays und Reden. Frankfurt (M.).

KLÜGER, RUTH (1992): weiter leben. Eine Jugend. Göttingen.

- (2006): Trauer um die Toten, aussichtsloses Überleben. In: Literaturen 10:52-53.

KöSTER, Juliane (2005): Bernhard Schlink. Der Vorleser. Interpretation. München/Düsseldorf/Stuttgart.

Krall, Hanna (1992): Dem Herrgott zuvorkommen. Aus dem Polnischen von Hubert Schumann. Frankfurt (M.).

KrZEMiŃski, AdAm (2005): So viele Kriege wie Nationen. Aus dem Polnischen von Silke Lent: www.societyofcontrol.com/library/war/krzeminski_kriege_und_Nationen. txt (29.12.08).

KULTUSMINISTERIUM NRW (1994): Lektüre von Ganzschriften im Fach Deutsch der Sekundarstufe 1 des Gymnasiums in NRW. Frechen.

LANDAU-CZAJKa, ANNA (im Gespräch mit Olga Wysocka) (2005): „Die Teilung in Gut und Böse beginnt sich zu verwischen “. In: KERSKI / OWCZAREK, 75-85.

LÜHE, IRMELA VON DER (1999): Interviews gegen das Verschweigen. Hanna Kralls Reportagen. In: EgGeRT, HARTMUt / GoleC, JANUSZ (eds.): „.... wortlos der Sprache mächtig " Schweigen und Sprechen in der Literatur und sprachlicher Kommunikation. Stuttgart, 95-110.

MajChrowski, ZBigniew / Rosiek, STANiSŁAW (2006): Między tekstami. Język polski. Część 5. [Zwischen den Texten. Polnische Sprache. Teil 5.]. Warszawa.

Makowiecki, ANdrzej Z. / Markowski, Andrzej / PASZYŃSki, WŁodZimierZ / WrocZYŃSKI, TOMASZ (2004): „Pamiętajcie o ogrodach...“ Kultura. Literatura. Język. Część 3. [„Erinnert euch an die Gärten...“ Kultur Literatur Sprache. Teil 3] Warszawa.

Meyer, MARITA (2008): Das Thema „Holocaust“ im Deutschunterricht: eine Kluft zwischen Literaturwissenschaft und schulischer Praxis? Ergebnisse einer Umfrage. In: Der Deutschunterricht 1:74-80.

MiŁosz, CzesŁaw (1974): Verführtes Denken. Aus dem Polnischen von Alfred Loepfe. Frankfurt (M.).

- (1999): Dar / Gabe. Ausgewählt und übertragen von Karl Dedecius. Kraków.

- (2003): Karussell. Aus dem Polnischen von Hans-Christian Trepte für Sample Translations: www.instytutksiazki.pl (5.7.2008).

MocZARSKI, KAZIMIERZ (1978): Gespräche mit dem Henker. Das Leben des SSGruppenführers und Generalleutnants der Polizei Jürgen Stroop aufgezeichnet im Mokotów-Gefängnis zu Warschau. Aus dem Polnischen von Margitta Weber. Düsseldorf. 
Deutsche und polnische Lektüren über den Zweiten Weltkrieg und den Holocaust

MüLleR-Michaels, HARRO (1994): Sichtung und Kommentierung der Ergebnisse der Umfrage zur Lektüre von Ganzschriften im Deutschunterricht der Gymnasien in der Sekundarstufe 1 in Nordrhein-Westfalen. In: KULTUSMINISTERIUM NRW: Lektüre von Ganzschriften im Fach Deutsch der Sekundarstufe 1 des Gymnasiums in NRW. Frechen, 54-60.

NaŁKowsKa, Zofia (1968): Medaillons. Aus dem Polnischen von Henryk Bereska. Frankfurt (M.).

OSTERMANN, MichA (2004): aporien des erinnerns - bernhard schlinks roman der vorleser. Bochum.

SAUERLAND, Karol (2004): Polen und Juden zwischen 1939 und 1968. Jedwabne und die Folgen. Berlin/Wien.

- (2007): Tadeusz Borowski, Celine und die Rezensenten. In: Fritz Bauer Institut. Newsletter 31:14-18.

Schlant, ERnestine (2001): Die Sprache des Schweigens. Die deutsche Literatur und der Holocaust. München

SCHLINK, BERNHARD (1995): Der Vorleser. Zürich.

SEghers, AnNa (1946/282007): Das siebte Kreuz. Ein Roman aus Hitlerdeutschland. Berlin.

WeISS, Peter (1965): Die Ermittlung. Oratorium in 11 Gesängen. Frankfurt (M.).

Welzer, Harald / Moller, SABine / Tschuggnall, Karoline (2003): „, Opa war kein Nazi “. Nationalsozialismus und Holocaust im Familiengedächtnis. Frankfurt (M.). 